\title{
Early time evolution of a chemically produced electron depletion
}

\author{
W. A. Scales \\ Bradley Department of Electrical Engineering, Virginia Tech, Blacksburg, Virginia \\ P. A. Bernhardt and G. Ganguli \\ Beam Physics Branch, Plasma Physics Division, Naval Research Laboratory, Washington, D.C.
}

\begin{abstract}
The early time evolution of an ionospheric electron depletion produced by a radially expanding electron attachment chemical release is studied with a twodimensional simulation model. The model includes electron attachment chemistry, incorporates fluid electrons, particle ions and neutrals, and considers the evolution in a plane perpendicular to the geomagnetic field for a low beta plasma. Timescales considered are of the order of or less than the cyclotron period of the negative ions that result as a by-product of the electron attachment reaction. This corresponds to time periods of tenths of seconds during recent experiments. Simulation results show that a highly sheared azimuthal electron flow velocity develops in the radially expanding depletion boundary. This sheared electron flow velocity and the steep density gradients in the boundary give rise to small-scale irregularities in the form of electron density cavities and spikes. The nonlinear evolution of these irregularities results in trapping and ultimately turbulent heating of the negative ions.
\end{abstract}

\section{Introduction}

Electron attachment materials such as $\mathrm{SF}_{6}$ have been utilized in ionospheric modification experiments for over 30 years [Mendillo and Forbes, 1982, and references therein]. In recent years, there has been renewed interest in the electron density depletions artificially produced by this type of chemical release. These artificial depletions have a wide variety of potential uses including perturbation of auroral current systems [Bernhardt et al., 1991] and focusing of high-power radio waves [Bernhardt et al., 1994]. Also, because of the negative ion plasma created as a chemical by-product of these releases, opportunity exists for studying the physics of negative ion and dusty plasmas in space [Ganguli et al., 1992]. The electron attachment release reduces the electron density directly through dissociative electron attachment. Attachment chemicals have been shown to be superior in many respects to chemicals classically used such as $\mathrm{H}_{2} \mathrm{O}$ and $\mathrm{H}_{2}$ which produce electron depletions indirectly through ion-molecule reactions [Bernhardt et al., 1991]. The attachment releases produce faster and larger perturbations because of larger reaction rate constants. Chemicals used during recent experiments include sulfur hexafluoride $\left(\mathrm{SF}_{6}\right)$, trifloromethyl bromide $\left(\mathrm{CF}_{3} \mathrm{Br}\right)$, and nickel carbonyl

Copyright 1995 by the American Geophysical Union.

Paper number 94JA02490.

0148-0227/95/94JA-02490\$05.00
$\left(\mathrm{Ni}(\mathrm{CO})_{4}\right)$. The corresponding dissociative electron attachment reactions for these chemicals are as follows

$$
\begin{gathered}
\mathrm{SF}_{6}+e^{-} \rightarrow \mathrm{SF}_{5}^{-}+\mathrm{F} \\
\mathrm{CF}_{3} \mathrm{Br}+e^{-} \rightarrow \mathrm{Br}^{-}+\mathrm{CF}_{3} \\
\mathrm{Ni}(\mathrm{CO})_{4}+e^{-} \rightarrow \mathrm{Ni}(\mathrm{CO})_{3}^{-}+\mathrm{CO}
\end{gathered}
$$

The attachment rate coefficients $k$ are typically of the order of $10^{-7} \mathrm{~cm}^{3} / \mathrm{s}$. The mass of the negative ions produced by these reactions is much larger than the mass of the dominant positive ion $\left(\mathrm{O}^{+}\right)$at the altitudes of interest. The release produces an initial plasma configuration in which the ambient ionospheric plasma $\left(e^{-}-\right.$ $\left.\mathrm{O}^{+}\right)$is separated from a negative ion plasma by a relatively thin boundary layer.

A series of recently conducted sounding rocket experiments, NICARE 1 [Bernhardt et al., 1991], NICARE 2 [Bernhardt et al., 1993], and CRRES ionospheric focused heating (IFH) [Bernhardt et al., 1994], have been sucessful at creating electron depletions with maximum diameters of up to $30 \mathrm{~km}$ by releasing attachment materials. These experiments were the first to make in situ measurements inside of electron depletions produced by attachment chemical releases. The measurements showed that the electron density was reduced from on the order of $10^{5}$ to $10^{2} \mathrm{~cm}^{-3}$ on timescales much less than the negative ion cyclotron period $(\sim 0.1 \mathrm{~s})$. High time resolution Langmuir probe measurements showed this electron density reduction to typically occur within several milliseconds. Initially, the boundary layer separating the ambient and negative ion plasma was quite 
thin with a thickness of the order of $100 \mathrm{~m}$ or less. Small-scale plasma irregularities in the form of electron density cavities and spikes with diameters of 1-10 m were observed in the boundary layer. The timescale for the irregularity development was less than the negative ion cyclotron period $(\sim 0.1 \mathrm{~s})$.

These experimental observations have prompted new theoretical and numerical simulation modeling efforts since past work had primarily considered the depletion evolution on large space and timescales. These early works considered electron attachment and neutralization chemistry, airglow production, macroscopic electrodynamics, and macroscopic plasma instabilities [Mendillo and Forbes, 1982; Bernhardt, 1984, 1986, 1987, 1988; Bernhardt et al., 1991; Scales and Bernhardt, 1991]. Our earlier theoretical and simulation work [Ganguli et al., 1992; Scales et al. 1992, 1994a] was the first attempt to consider the early time microscopic processes associated with chemically produced electron depletions. The simulation work of Scales et al. $[1992,1994$ a] considered the evolution on timescales of the order of or less than the negative ion cyclotron period and spacescales of roughly 10 negative ion Larmor radii. This corresponds to timescales of tenths of seconds or less and spacescales of a few hundred meters or less during the experiments. The contribution of Scales et al. [1992, 1994a] was a preliminary description of the initial electrodynamic evolution of the depletion and the nonlinear development of plasma processes that occur in the depletion boundary layer. The principal results of this work showed that (1) a highly sheared electron flow velocity develops in the depletion boundary layer initially and (2) this sheared electron flow velocity acts as a free energy source for the development of waves in the boundary layer. Strongly sheared electron flows originate due to the density gradients at the boundary, especially since the gradients in the ion and electron densities are in opposite directions. This feature leads to physics which is unique to electron attachment chemical release experiments and distinguishes them from other chemical release experiments, such as the barium releases during the AMPTE series of experiments [Bernhardt et al., 1987; Winske, 1989; Huba et al., 1990]. As explained in Ganguli et al. [1992], the electron flow velocity transverse to the geomagnetic field $B$ at early times after release may be approximated by

$$
V_{\mathrm{E}}(r) \approx \frac{\mathrm{E}(r)}{\mathrm{B}}\left\{1+\left[\frac{\mathrm{T}_{e}}{\mathrm{~T}_{+}} n_{+}(r)+\frac{\mathrm{T}_{e}}{\mathrm{~T}_{-}} n_{-}(r)\right] \frac{1}{n_{e}(r)}\right\}
$$

where $n_{e}, n_{+}, n_{-}$and $T_{e}, T_{+}, T_{-}$are the electron, positive ion, and negative ion densities and temperatures, respectively. The sheared electric field $\mathrm{E}(r)$ develops in order to maintain quasi-neutrality at the boundary since the electrons are strongly magnetized and the negative ions are essentially unmagnetized on these timescales [Ganguli et al., 1992; Scales et al., 1994a]. Since $n_{e} \rightarrow 0$ in the electron depletion, it can be seen from (1) that strongly sheared electron flows may exist in the boundary layer. Waves develop and produce irregularities in the boundary layer because of the shear-driven electron-ion-hybrid instability (EIH) as described by Ganguli et al. [1992] and Scales et al. [1994a]. These works argued that the EIH instability should be the dominant source of irregularities rather than the lower hybrid drift (LHD) instability which has been proposed to explain irregularities observed during the AMPTE barium releases [Winske, 1989]. The fact that (1) the shear frequency (ratio of maximum flow velocity to flow velocity scale length) exceeds the lower hybrid frequency and (2) vortex structures are observed in the simulations support the EIH rather than LHD mechanism as is described by Romero et al. [1992b].

A limitation of our previous simulation work [Scales et al., 1992, 1994a] was that it did not include electron attachment chemistry and therefore could not realistically describe the creation and evolution of the electron depletion and negative ion plasma from the expanding neutral cloud. The present work has several purposes. The first is to extend the work of Scales et al. [1992, 1994a] by including attachment chemistry in the simulation model. This will provide a description of the overall early time (less than the negative ion cyclotron period) evolution of a chemically produced electron depletion as well as a more detailed and realistic description of the boundary layer processes than provided in the past work. The recent CRRES IFH experiment has provided high time resolution observations of small-scale plasma irregularities that exist in the depletion boundary layer [Bernhardt et al., 1994]. Recently, Scales et al. [1994b] have made a preliminary study of these irregularities by using our new simulation model. Therefore a second purpose of this work is to provide a more detailed study of these irregularities and the associated processes in the depletion boundary layer that may be used to aid in interpretation of the recent experimental measurements. This work is organized as follows. Section 2 provides a detailed description of our new simulation model. A representative simulation run is presented in section 3 . This section will consider the overall electrodynamics of the release as well as a study of wave generation and accompanying wave-particle interaction processes. Section 4 provides a summary and discussion of the results.

\section{Simulation Model}

We have developed a periodic two-dimensional electrostatic hybrid simulation model to study the evolution of chemically produced electron depletions. As in our previous particle-in-cell (PIC) model [Scales et al., 1992,1994 a], we consider a two-dimensional plane $(x y)$ perpendicular to the direction of the constant background magnetic field $\mathbf{B}$ denoted by $\hat{\mathbf{z}}$. Our hybrid model treats the electrons as a massless fluid while treating the ion species and neutrals as simulation particles. The validity of the fluid approximation for electrons has been verified from the results of our full PIC model simulations since the electron behavior remained fluidlike. The hybrid model allows us to efficiently in- 
corporate electron attachment chemistry. This chemistry is essential since electron attachment by the expanding neutral cloud causes the depletion boundary to expand in time. Our previous simplified model considered the case of a stationary boundary.

In our hybrid model, the ions are advanced in time by using the same standard PIC techniques [Hockney and Eastwood, 1988; Tajima, 1989; Birdsall and Langdon, 1991] as in our previous work [Scales et al., 1992, 1994a]. We consider two ion species as before, background $\mathrm{O}^{+}$and the negative ions $\mathrm{X}^{-}$, which are created through electron attachment by the neutrals. The $i$ th ion simulation particle is advanced in time from the full equations of motion

$$
\begin{gathered}
\frac{d \mathbf{x}_{i}}{d t}=\mathbf{v}_{i} \\
\frac{d \mathbf{v}_{i}}{d t}=\frac{q_{\mathrm{B}}}{m_{\mathrm{s}}}\left(\mathbf{E}+\mathbf{v}_{i} \times \mathbf{B}\right)
\end{gathered}
$$

Here $x_{i}$ and $v_{i}$ are the position and velocity of a particular ion and $q_{\mathrm{s}}$ and $m_{\mathrm{s}}$ denote the charge and mass of the ion species. The electric field $\mathbf{E}$ is evaluated at the particle positions by interpolation from the simulation grid points [Birdsall and Langdon, 1991]. The ion particles move in two-dimensional coordinate and velocity space.

In the hybrid model, we consider massless, fluid electrons that evolve in time $t$ according to the continuity and momentum equations

$$
\begin{gathered}
\frac{\partial n_{e}}{\partial t}+\mathbf{v}_{e} \cdot \nabla n_{e}=L \\
\mathbf{v}_{e}=\frac{\mathbf{E} \times \mathbf{B}}{B^{2}}
\end{gathered}
$$

Here $n_{e}$ and $v_{e}$ are the electron fluid density and velocity. The term $L$ is a loss term due to electron attachment which will be described in more detail shortly. Since the electrons are massless and have zero temperature, their velocity is simply given by the divergenceless $\mathbf{E} \times \mathbf{B}$ drift. The electric field is related to the electric potential by $\mathbf{E}=-\nabla \phi$. The continuity equation is solved by using the pseudospectral technique [Orszag, 1971]. The spatial dependence in (4) is decomposed into its spectral representation by using discrete Fourier transforms. This reduces the partial differential equation in (4) into the following ordinary differential equation

$$
\frac{d n_{e \mathbf{k}}}{d t}+\frac{1}{B} \sum_{\mathbf{p}+\mathbf{q}=\mathbf{k}}(\mathbf{p} \times \hat{\mathbf{z}}) \cdot \mathbf{q} \phi_{\mathbf{p}} n_{e \mathbf{q}}=L_{\mathbf{k}}
$$

where we have used (5) and written this expression in terms of the electric potential. This is the governing equation for the Fourier coefficients $n_{e k}$ of the electron density, where $\mathbf{k}$ denotes the Fourier mode number. The convolution term on the left-hand side of (6) is calculated in $\mathbf{x}$ space and dealiased by using the grid (or phase) shifting technique of Patterson and Orszag
[1971]. The coefficients are advanced in time by using a leapfrog method. This time integration scheme was shown to be strongly stable for sufficiently small time steps by Gottlieb and Orszag [1977].

The electric potential is calculated from the electron fluid density and the ion particle densities by using the Poisson equation

$$
\epsilon_{0} \nabla^{2} \phi=-q_{e} n_{e}-\sum_{\mathbf{s}} q_{i \mathrm{~s}} n_{i \mathrm{~s}}
$$

where $\epsilon_{0}$ is the permittivity constant and $q_{e}$ and $q_{i s}$ are the electron and ion charges. The second term on the right-hand side of (7) represents the ion contribution to the charge density with the subscript $s$ denoting the particular ion species. The ion density is calculated by counting the ions at the grid cells with an appropriate weighting as described by Birdsall and Langdon [1991]. Poisson's equation is solved in Fourier space as

$$
\epsilon_{0} k^{2} \phi_{\mathbf{k}}=q_{e} n_{e \mathbf{k}}+\sum_{\mathbf{s}} q_{i \mathrm{~s}} n_{i \mathbf{s k}}
$$

The neutrals are treated as simulation particles just as the ions. Each of the neutrals is given an initial position $\mathbf{x}_{0}$ and radially directed $(\hat{\mathbf{r}})$ velocity $\mathbf{v}_{0}$, and assumed to have a small thermal velocity $v_{\mathrm{X}}$ at $t=0$. All neutrals initially lie at the center of the simulation box (the release point) within a cylindrical disk of radius $r_{0}$ and the neutral density is constant within this region. Spatially, the radial velocity of neutrals is taken to vary from zero at the cloud center to a maximum value at the edge to be representative of the diffusive expansion of neutrals into the background plasma. That is, $\mathbf{v}_{0}=v_{r}\left(r / r_{0}\right) \hat{r}$, where we refer to $v_{r}$ as the neutral expansion velocity. The neutrals experience no force from the electric and magnetic fields and therefore the position of the $i$ th neutral as a function of time is

$$
\mathbf{x}_{i}(t)=\mathbf{v}_{0 i} t+\mathbf{x}_{0 i}
$$

In general, the electron attachment loss $L$ is given by

$$
L=-k n_{e} n_{\mathrm{n}}
$$

where $n_{\mathrm{n}}$ is the neutral density and $k$ is the attachment rate coefficient. Both the neutral and electron density loss rates are governed by this expression [Scales and Bernhardt, 1991, and references therein]. A model can be specified for the neutral particle number variation with time due to the electron attachment. We consider a simplified model in which the total number of neutral particles $N_{\mathrm{n}}$ varies with time $t$ as

$$
N_{\mathrm{n}}(t)=N_{\mathrm{n} 0} \exp (-t / \tau)
$$

where $N_{\mathrm{n} 0}$ is the total number of neutrals at $t=0$, and $\tau$ is the neutral particle attachment time constant. The constant $\tau$ is choosen to produce electron density reduction rates that are comparable to those observed during recent experiments as will be described shortly. It should be noted that since the number of simulation 
neutrals is much less than during actual experiments, the simulation $\tau$ must be artificially small compared to the physical value $\tau=\left(k n_{e}\right)^{-1}$ to produce the desired density reductions.

In the simulation, we adopt the following simplified model for the attachment chemistry which incorporates the essential aspects and is computationally feasible and efficient. Each time step $\Delta t$, a number of neutrals (determined by (11)) are randomly converted into negative ions in regions of nonzero electron density. These negative ions retain the same position, velocity, and mass as the neutral. If an attempt to produce a negative ion in a region of zero electron density is made, the "attachment" is prohibited and the neutral is allowed to make another attempt at a later time step. Instead of calculating the loss term $L$ from (10) directly it is more computationally efficient to calculate $L$ by using the fact that the negative ion production is $-L$. Therefore each time step, the density of the newly created negative ions is calculated as described earlier with the appropriate grid weighting. Denoting this density by $\Delta n_{\mathrm{X}}$, the loss term $L$ in (4) is calculated from

$$
L=-\frac{\Delta n_{\mathbf{X}}}{\Delta t}
$$

Note that our model does not directly take into account the fact that the electron attachment loss rate decreases with decreasing electron density as is described by (10). We have developed a more sophisticated model which incorporates this process within the framework of the simplified model just described, however, we find that the results to be qualitatively the same.

The initial configuration of the simulation we present in the next section is as follows. The simulation box size is $128 \Delta \times 128 \Delta$, where $\Delta$ denotes the grid spacing. The Larmor radius of negative ions moving at the expansion velocity $v_{r}$ is denoted by $\rho_{\mathrm{X}}$ and $\rho_{\mathrm{X}}=16 \Delta$. In terms of $\rho_{\mathrm{X}}$, the simulation box size is $8 \rho_{\mathrm{X}} \times 8 \rho_{\mathrm{X}}$. The initial density of the ambient positive ions is constant and equal to 30 particles per grid cell $\left(30 \Delta^{-2}\right)$. Quasineutrality requires that the initial electron fluid density be set equal to this value. At early times after release, the neutral density is much larger than the background plasma density. In the simulation, the initial neutral cloud radius $r_{0}$ is taken to be $4 \Delta$ and the neutral density 6000 particles per grid cell $\left(6000 \Delta^{-2}\right)$. This represents the initial impulselike release of the neutrals into the ambient plasma. The radial expansion velocity $v_{r}$ is choosen so $v_{r}=20 v_{\mathrm{X}}=0.25 v_{0}$, where $v_{\mathrm{X}}$ and $v_{\mathrm{O}}$ are the thermal velocities of the negative ions (and neutrals) and positive ions. These values were choosen to model conditions during the experiments where the expansion velocity and $\mathrm{O}^{+}$thermal velocity are roughly estimated to be 300 and $1200 \mathrm{~m} / \mathrm{s}$ respectively. Since the neutrals are expected to have a relatively small thermal velocity we take $v_{\mathbf{X}} \ll v_{r} \ll v_{0}$. The mass ratio of the negative and positive ions is 8:1 which is representative of the ratio for the negative ions described earlier and $\mathrm{O}^{+}$. The time period over which the run is made is $\Omega_{\mathrm{X}} t=3$, where $\Omega_{\mathrm{X}}$ is the negative ion cyclotron (radian) fre- quency and $\tau_{X}=2 \pi / \Omega_{X}$ is the negative ion cyclotron period. As stated earlier, recent experimental observations show reductions in the electron density by a factor of $10^{3}$ on timescales of the order of $0.001 \mathrm{~s}$ which corresponds to roughly $0.01 \tau \mathrm{X}$ for the attachment chemicals under consideration. The electron density reduction in the simulation model can be no more than a factor of 30 for an initial electron density of $30 \Delta^{-2}$. During the experiments, it follows that a reduction by a factor of 30 occurs in roughly $0.005 \tau_{X}$. In the simulation, the neutral particle attachment time constant $\tau$ is choosen such that $\Omega_{\mathbf{X}} \tau=3$ which produces a comparable electron density reduction rate in the simulation. This can be seen as follows. The electron density reduction rate early in the simulation $(t \ll \tau)$ can be estimated from $d n_{e} / d t \approx-n_{\mathrm{n}} / \tau$. Using the simulation values, we find $d n_{e} / d t \approx-10^{4} \Delta^{-2} \tau_{\mathbf{X}}^{-1}$. This corresponds to a reduction of $30 \Delta^{-2}$ in $0.003 \tau \mathrm{x}$.

To scale our simulation space and timescales to the experiments, we need to consider the negative ion cyclotron period and Larmor radius. As we stated earlier, the typical cyclotron period for the negative ions at the altitudes of interest is a few tenths of a second. For negative ions moving at an expansion speed of several hundred meters per second, $\rho_{X}$ will be of the order of $10 \mathrm{~m}$. Therefore our model considers spacescales of the order of $100 \mathrm{~m}$ and timescales on the order of $0.1 \mathrm{~s}$. We believe that neglecting ion-neutral collisions is a reasonable approximation in our model since Scales and Bernhardt [1991] estimate this collision time to be of the order of $1 \mathrm{~s}$.

\section{Results}

We now present the results of a representative run of the simulation model described in the previous section. The discussion will consist of two parts. The first is a discussion of the initial creation of the electron depletion and negative ion plasma as well as the initial electrodynamic evolution. This will be followed by a description of the development of irregularities in the depletion boundary layer.

\subsection{Depletion and Negative Ion Cloud Creation}

Figure 1 shows the initial and final radial density of the neutral cloud. As stated earlier, the initial neutral cloud radius is $4 \Delta$ and the density is 6000 particles per grid cell to model the impulselike release. By the end of the simulation, the neutral density has been reduced to 30 particles per cell due to radial expansion and electron attachment. The neutral density at the end of the run is of the order of the initial background plasma density. Figure 2 shows the radial drift velocity of the neutrals and the two ion species averaged over all particles in the simulation box. Note that the negative ion cloud slows down relative to the expanding neutral cloud. The positive ions acquire a negligibly small radial drift velocity. The average neutral velocity in Figure 2 decreases with time since the faster neutrals populate the expanding 


\section{NEUTRAL DENSITY}

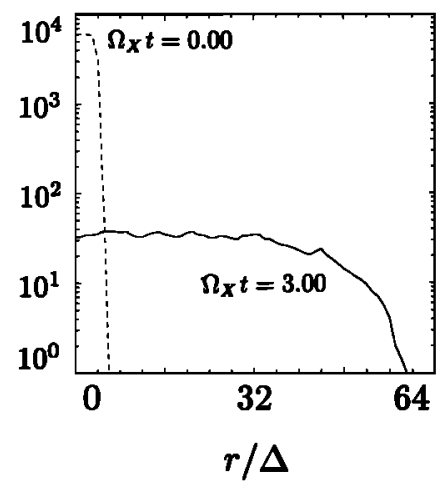

Figure 1. Neutral cloud density (in particles per cell) at the beginning and end of the simulation.

depletion boundary where they are lost to electron attachment.

The radial density of the three plasma species is shown at two times, $\Omega_{\mathrm{X}} t=0.25$ and 2.5, in Figure 3 . The electron density has already been reduced to nearly zero inside the depletion at $\Omega_{\mathrm{X}} t=0.25$ and a sharp electron density gradient exists at the depletion boundary. The negative ions are created with the expansion velocity of the neutral cloud. Since the negative ions are unmagnetized on this timescale, they are free to move radially across the magnetic field unlike the electrons which only azimuthally $\mathbf{E} \times \mathbf{B}$ drift. Therefore, initially, there is a pile-up of negative ions inside the radially expanding depletion boundary which can be seen at $\Omega_{\mathrm{X}} t=$ 0.25 . This negative charge buildup at the boundary produces a strong radial electric field $E_{r}$. This ambipolar electric field accelerates the ambient positive ions away from the release point and produces a depression in the positive ion density. This result was also noted in our previous work [Ganguli et al., 1992; Scales et al., 1994a] and is characteristic of the electrostatic snowplow effect described by Bernhardt et al. [1991]. At $\Omega_{\mathrm{X}} t=2.5$, the electron depletion boundary has expanded to a radius of

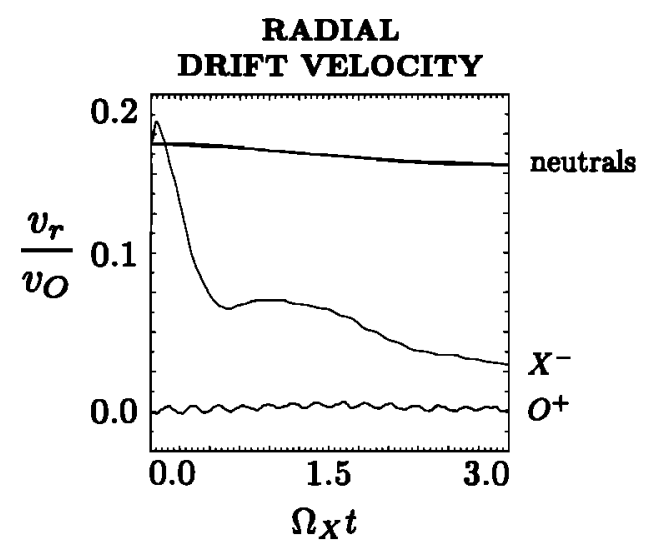

Figure 2. Radial drift velocity (averaged over all particles) for the neutrals and ions during the simulation. Note that the negative ion cloud slows down relative to the neutral cloud.

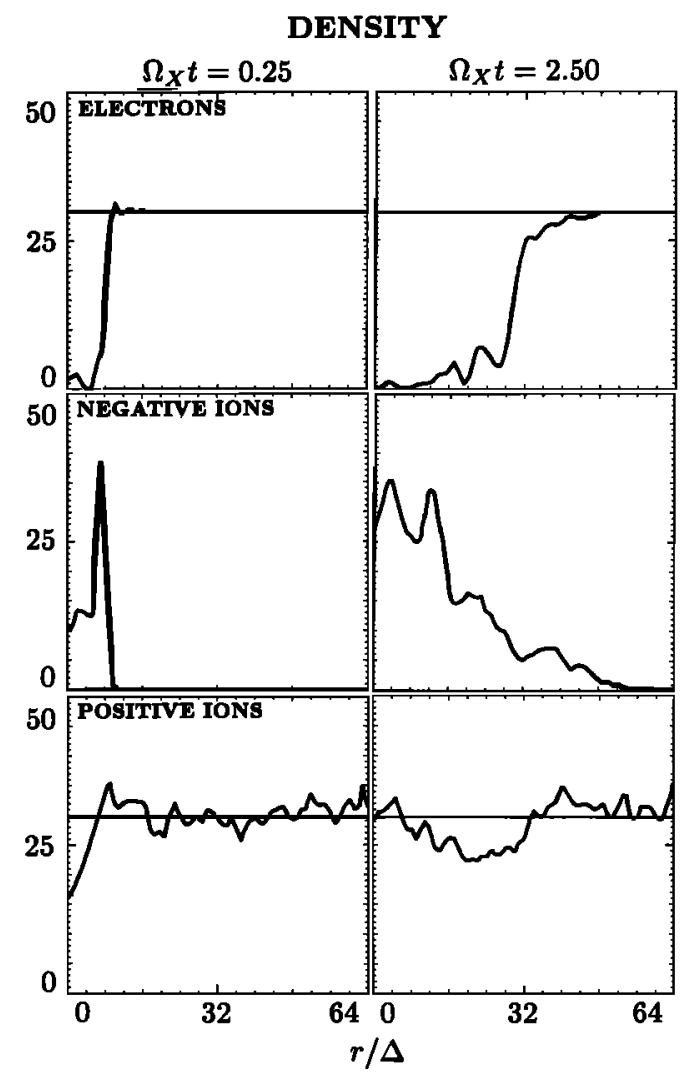

Figure 3. Radial density of the three plasma species at $\Omega_{\mathrm{X}} t=0.25$ and 2.50. Dashed line indicates values at $\Omega_{\mathrm{X}} t=0$. Note radial expansion of the depletion boundary.

roughly $32 \Delta$. The boundary has broadened somewhat, but the electron density gradient is still relatively steep.

The large radial electric field across the boundary layer produces an azimuthal $\mathbf{E} \times \mathbf{B}$ flow in the plasma. Since the ions are essentially unmagnetized on these timescales, the $\mathbf{E} \times \mathbf{B}$ flow of the ions is small in comparison to the flow of electrons as was observed in the simulations of Scales et al. [1994a] and predicted by the theoretical calculations of Ganguli et al. [1992]. The electron azimuthal flow velocity $\left(V_{\mathrm{E}}\right)$ is shown in Figure 4 at $\Omega_{\mathrm{X}} t=0.25,1.50,3.00$. At $\Omega_{\mathrm{X}} t=0.25$, the electron flow velocity is highly sheared and localized at the depletion boundary with a value of $V_{\mathrm{E}} \approx-1.6 v_{\mathrm{O}}$. (The boundary is at $r \approx 10 \Delta$ at $\Omega_{\mathrm{X}} t=0.25$ as shown in Figure 3.) As time evolves to $\Omega_{\mathrm{X}} t=1.5$, the boundary layer radially expands and the maximum electron flow velocity is coincident with the depletion boundary which is roughly at $20 \Delta$. The maximum value of the flow velocity has decreased somewhat to $-1.25 v_{0}$. At the end of the simulation, $\Omega_{\mathrm{X}} t=3.0$, the flow velocity has relaxed significantly. As will be discussed later, this flow velocity is reduced because of the development of irregularites which broaden the boundary layer.

Figure 5 shows the temporal development of the electrostatic field energy (in the whole simulation box) during the simulation. The radial and azimuthal field energies are shown separately with the total field energy 


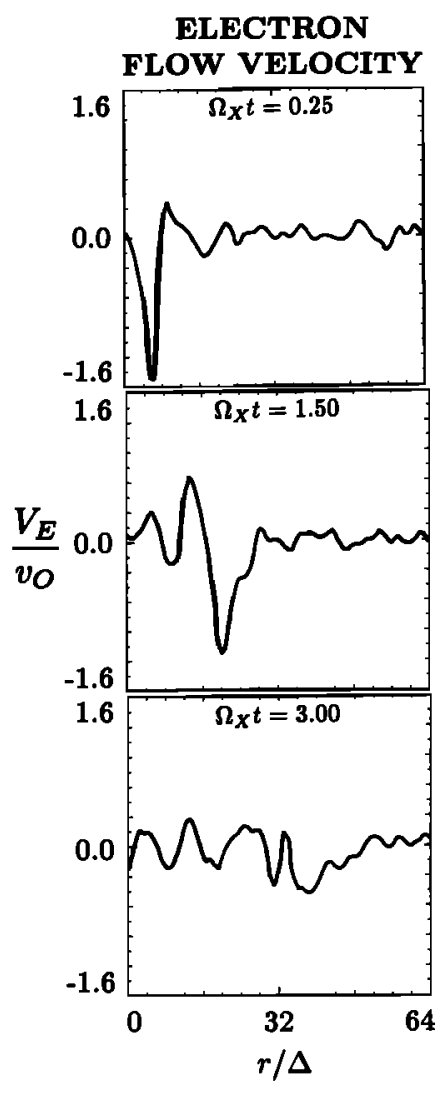

Figure 4. Electron flow $(\mathbf{E} \times \mathbf{B})$ velocity at $\Omega_{\mathrm{X}} t=$ $0.25,1.50$, and 3.00. Note that maximum flow is in the depletion boundary and the velocity profile relaxes by the end of the simulation.

equal to their sum. The radial field results from the negative charge surplus at the boundary as has been previously discussed. The azimuthal field results from azimuthally propagating electrostatic waves driven by plasma instabilities that will be discussed shortly. The radial field energy increases rapidly, reaches a maximum value at roughly $\Omega_{\mathrm{X}}=0.75$, and then remains at roughly a constant value. The reason that the radial field energy remains near a constant value is that

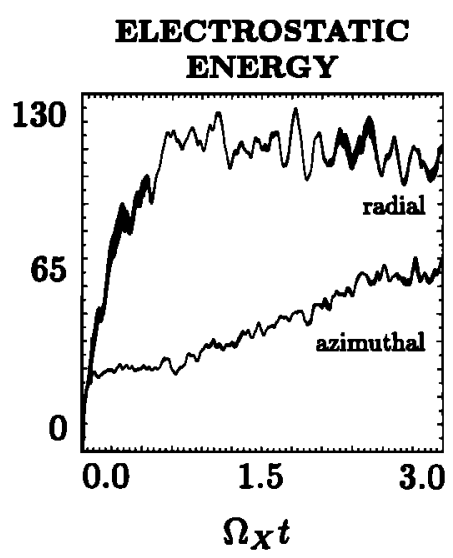

Figure 5. Radial and azimuthal electrostatic field energy during the simulation. the chemistry sustains the relatively sharp density gradient and negative charge surplus at the boundary. It should be noted that this was not the case in the work by Scales et al. [1994a] since that work did not include attachment chemistry. In that work, anomalous diffusion of the electrons into the depletion caused by waves reduced the negative charge surplus, relaxed the sharp electron density gradient, and produced a decay in the field energy as the system evolved in time. Also, in Figure 5, we see that near the time the radial energy reaches a maximum value, the azimuthal field energy begins to grow above the noise level and continues to grow until roughly $\Omega_{\mathrm{x}} t=2.5$. The azimuthal energy is the result of unstable plasma waves in the boundary layer. These waves produce irregularities which are the subject of the next section.

\subsection{Depletion Boundary Layer Irregularities}

To display the development of the boundary layer irregularities in more detail, Figure 6 shows the twodimensional density of the three plasma species at three times during the simulation. At $\Omega_{\mathrm{X}} t=0.25$, the electron depletion and negative ion plasma have just been completely formed. The gradients at the boundary are very steep and irregularities in the density are barely visable though they exist. At $\Omega_{\mathrm{X}} t=1.25$, well-defined structures are observed in the electron density. Also, structures are beginning to become more evident in the negative ion cloud as well. At $\Omega_{\mathrm{x}} t=2.5$, irregularities are more evident as well as vortex development in the electron depletion boundary layer. This structuring ultimately broadens the boundary layer. Irregularities are also evident in the negative ion cloud in the form of density clumps. It should be noted that the work of Scales et al. [1992, 1994a] showed negligible structuring of the negative ion cloud. The accompanying electrostatic potential $\phi$ is shown in Figure 7. The potential shows the development of irregularities as well. At the end of the simulation, the potential, like the boundary layer, exhibits broadening in space.

Azimuthal cross sections of the plasma density and potential at $\Omega_{\mathrm{X}} t=1.75$ are shown in Figures 8 and 9 . The cross sections are shown at three radii. The three radii $r / \Delta=30,26$, and 22 correspond to cross sections (1) taken outside the depletion, (2) at the outer edge of the depletion boundary, and (3) at the inner edge of the depletion boundary, respectively. At $r / \Delta$ $=30$, the plasma density is uniform with no evidence of irregularities. At $r / \Delta=26$, the electron density exhibits deep density cavities. Coincident with the electron density cavities are enhancements in the negative ion density. These correspond to the density clumps observed in Figure 6. At the inner edge of the depletion boundary, the electron density irregularities exhibit more spike-like signatures. The electron density cavities and spikes in Figure 8 show many similarities with the experimental observations during the CRRES IFH experiment [Bernhardt et al., 1994]. Irregularities are evident in the negative ion density. At both positions inside the depletion boundary, there is some evidence 


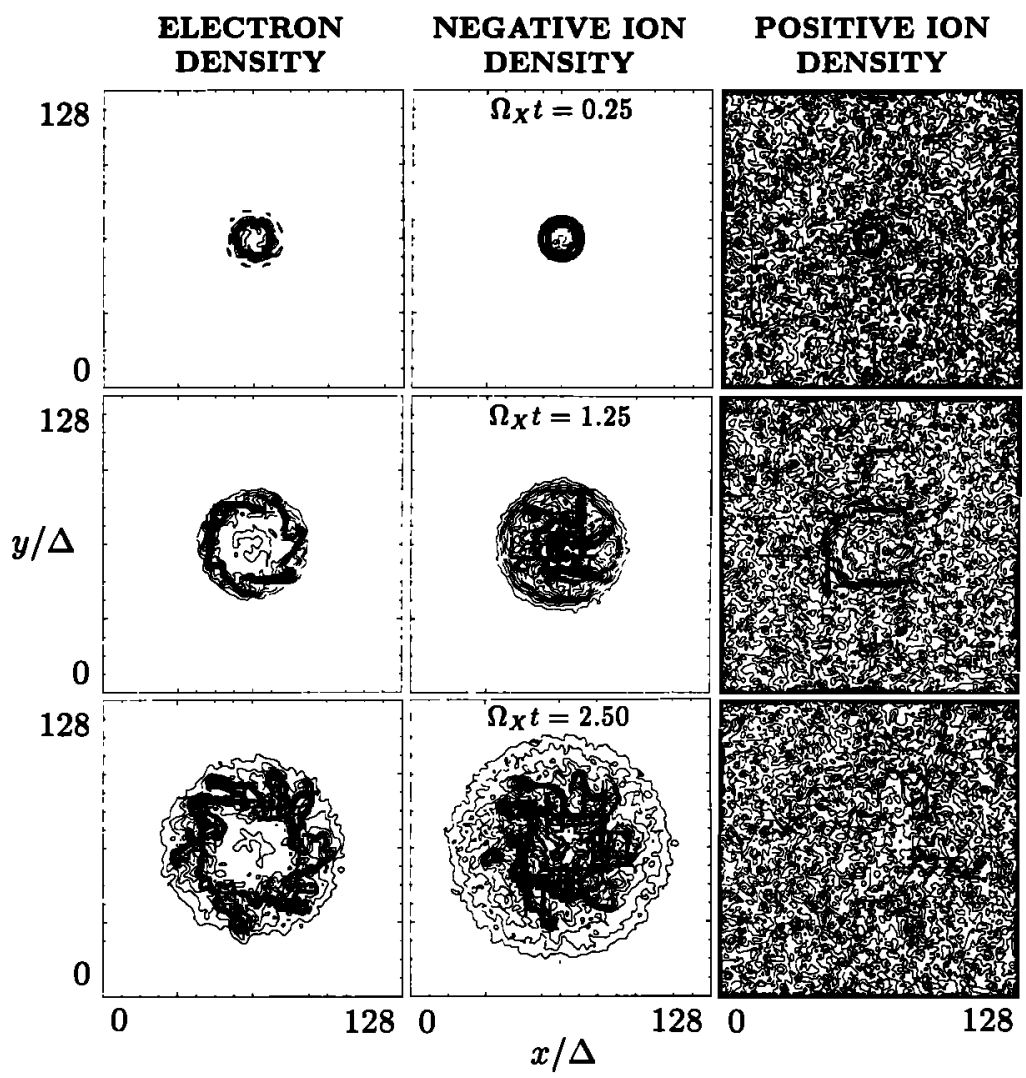

Figure 6. Two-dimensional density of the three plasma species at $\Omega_{\mathrm{X}} t=0.25,1.25$, and 2.50 . Note irregularity development in the electron and negative ion cloud boundaries.

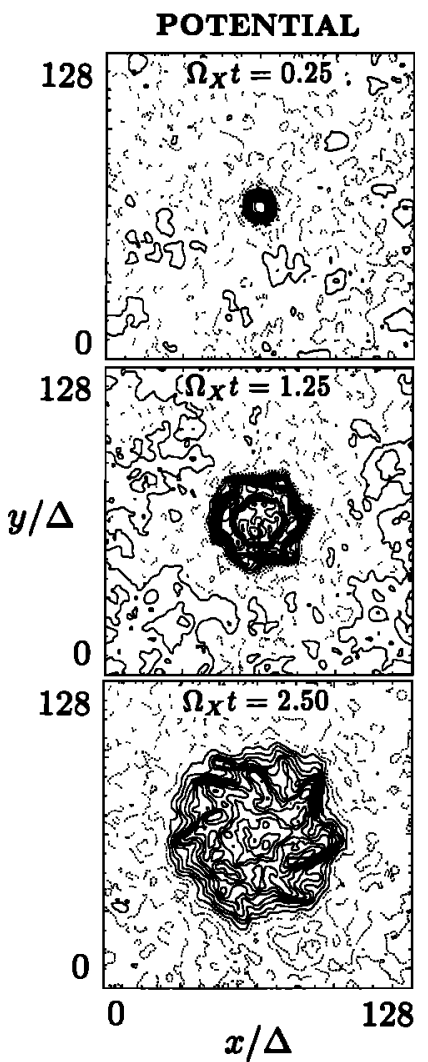

Figure 7. Electrostatic potential at $\Omega_{\mathrm{X}} t=0.25,1.25$, 2.50. Again, note irregularity development. of small cavities in the positive ion density. The potential shown in Figure 9 exhibits relatively deep potential wells associated with the electron density irregularities. This large potential ultimately traps the negative ions.

Figure 10 shows the temporal evolution of the azimuthal thermal energy (averaged over all particles) for the positive and negative ions, $T_{\mathrm{O}}$ and $T_{\mathrm{X}}$ respectively. The positive ion energy increases negligibly during the simulation. Most of the wave-particle heating is in the negative ions where the energy increases by a factor of roughly 90 . The negative ion energy increases until $\Omega_{\mathrm{x}} t \approx 2.5$ which is when the azimuthal electrostatic field energy in Figure 5 saturates. Since $v_{0}=80 v_{\mathbf{X}}$ initially, $T_{X}<T_{0}$ at the end of the run, however.

The azimuthal phase space at the fixed radius $r / \Delta$ $=26$ is shown in Figure 11 at three times, $\Omega_{\mathrm{X}} t=1.25$, 1.75 , and 2.75. This radius corresponds to one of the same values shown Figures 8 and 9 so comparisons can be made. At $\Omega_{\mathrm{X}} t=1.25$, the phase space shows negative ions that are near the leading edge of the expanding neutral cloud. Perturbations are barely evident. However, a small positive azimuthal flow velocity $v_{\theta \mathrm{X}}$ exists in the negative ions which is consistent with our earlier results [Ganguli et al., 1992; Scales et al., 1992, 1994a]. At $\Omega_{\mathrm{X}} t=1.25, v_{\theta \mathrm{X}} \approx 7 v_{\mathrm{X}}$. Later, at $\Omega_{\mathrm{X}} t=$ 1.75 , the phase space shows the negative ions at the outer edge of the expanding depletion boundary layer. Trapping is evident and hot negative ions can be seen to be coincident with the electron density cavities seen 


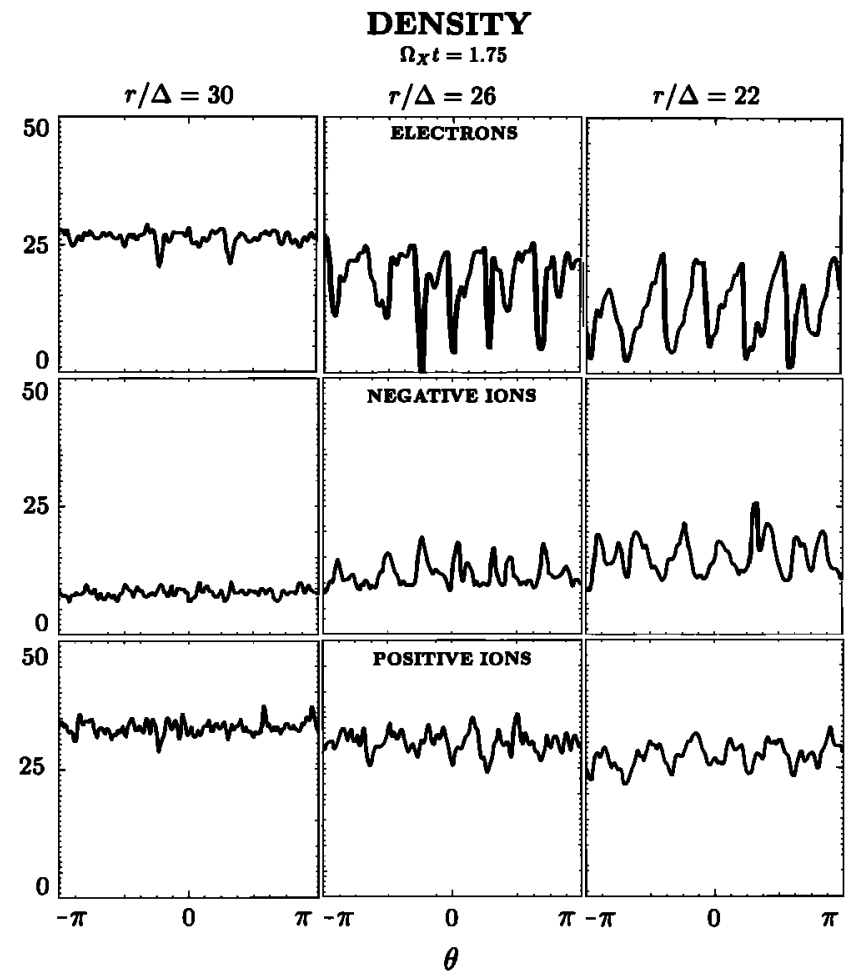

Figure 8. Azimuthal cross sections of the density for the three plasma species at $\Omega_{\mathrm{X}} t=1.75$. Note $r / \Delta=$ 30,26 , and 22 corresponds to outside the depletion, the depletion boundary outer edge, and the boundary inner edge, respectively. Note irregularities in the form of density cavities and spikes.

in Figure 8. Also, from Figure 9, it is seen that these ions are trapped in the steep potential wells associated with these irregularities. At late time, $\Omega_{\mathrm{X}} t=2.75$, the phase space shows that negative ions inside the depletion have been strongly scattered in phase space with some particles reaching energies near $30 v_{\mathrm{X}}$. Therefore the particles in the tail of the distribution reach energies near that of the background $\mathrm{O}^{+}$. The wave amplitude increases until the potential becomes sufficiently large to trap the negative ions. In this case, the condition on the potential for trapping is [Hasegawa, 1975]

$$
e \phi \sim \frac{1}{2} m_{X}\left(v_{\theta X}-v_{\phi}\right)^{2}
$$

where $e$ is the unit charge and $v_{\phi}=\omega / k$ is the wave phase velocity. We estimate $v_{\phi} \approx-15 v_{\mathrm{X}}$ from the perturbations in the negative ion phase space. Using this estimate for $v_{\phi}$ and our estimate for $v_{\theta \mathrm{X}}$ implies $e \phi / T_{\mathrm{X}} \sim 240$ for trapping which is in agreement with Figure 9. The fact that $v_{\phi} \varsigma v_{\theta \mathrm{X}} \ll v_{\mathrm{O}}$ explains why heating is primarily observed in the heavy negative ions rather than the lighter positive ions. Most of the negative ions are near resonance with the waves for this $v_{\phi}$.

Scales et al. [1994a] argued that the electron-ionhybrid (EIH) instability [Ganguli et al., 1988; Romero et al., 1992a] was the source of the waves in the depletion boundary layer observed in their simulations. The free

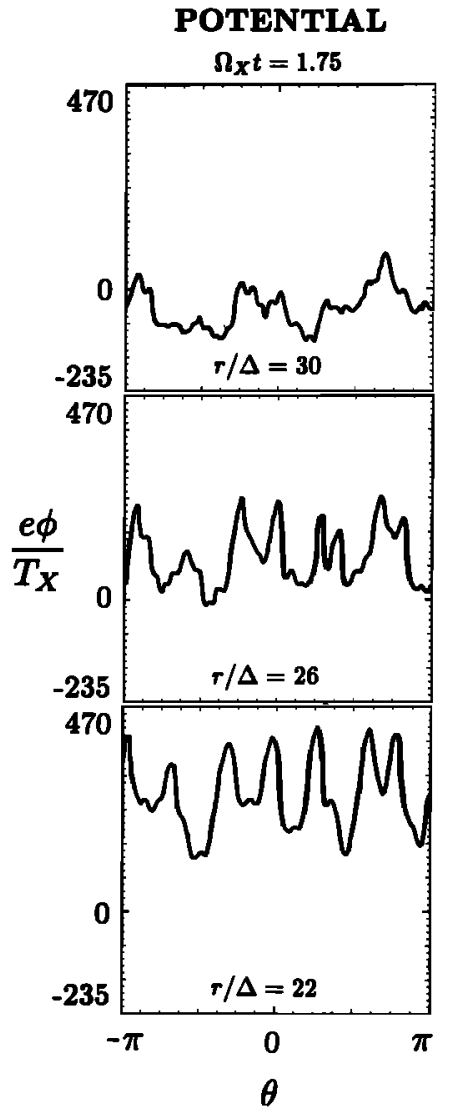

Figure 9. Azimuthal cross sections of the electrostatic potential at $\Omega_{\mathrm{X}} t=1.75$. The radii shown are the same as in Figure 8. Note the potential wells in the boundary layer.

energy for this instability comes from the highly sheared electron flow velocity $V_{\mathrm{E}}$ in the boundary. This instability produces waves with linear growth near the lower hybrid frequency $\omega_{\mathrm{LH}}$ where $\omega_{\mathrm{LH}}^{2}=\omega_{\mathrm{o}}^{2} /\left(1+\left(\omega_{\mathrm{pe}}^{2} / \Omega_{\mathrm{ce}}^{2}\right)\right)$ and $\omega_{0}, \omega_{\text {pe }}$, and $\Omega_{\text {ce }}$ are the $\mathrm{O}^{+}$plasma frequency and the electron plasma and cyclotron frequencies respectively. The nonlocal fluid dispersion relation for

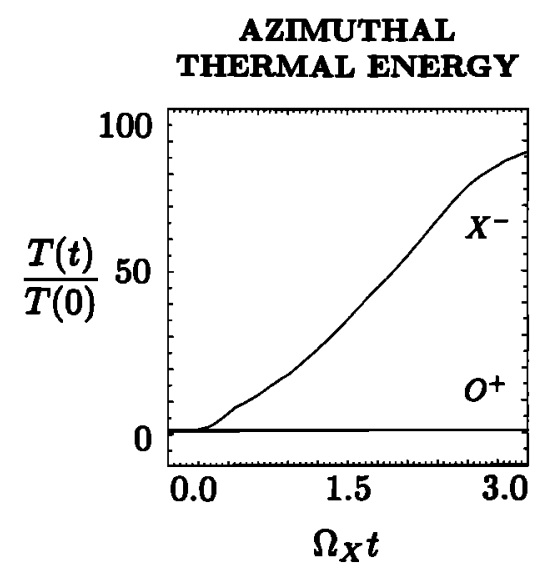

Figure 10. Azimuthal thermal energy (averaged over all particles) for the ions during the simulation. Note negative ion heating. 
frequency $\omega$ and wavenumber $k_{y}$ along the direction of the flow velocity is given by Ganguli et al. [1992] as

$$
\left\{\frac{d^{2}}{d x^{2}}-k_{y}^{2}-G(\omega) \frac{k_{y}\left(V_{\mathrm{E}}^{\prime \prime}(x)-\epsilon_{\mathrm{n}} \Omega_{\mathrm{ce}}\right)}{\omega-k_{y} V_{\mathrm{E}}(x)}\right\} \tilde{\phi}_{1}(x)=0
$$

where $G(\omega)=\delta^{2} /\left(\delta^{2}+1\right)\left(1-\left(\omega_{\mathrm{LH}} / \omega\right)^{2}\right), \delta=\omega_{\mathrm{pe}} / \Omega_{\mathrm{ce}}$, $V_{\mathrm{E}}^{\prime \prime}=d^{2} V_{\mathrm{E}} / d x^{2}$, and $\epsilon_{\mathrm{n}}=\left(d n_{e} / d x\right) / n_{e}=1 / L_{\mathrm{n}}$ is the inverse density gradient scale length. The perturbed potential is given by $\tilde{\phi}_{1}(x)$. In the simulation geometry, $r$ and $\theta$ correspond to $x$ and $y$, respectively, in the above expression. In our simulation limit, $m_{e} \rightarrow 0$. This implies $\delta \rightarrow 0$ and $\omega_{\mathrm{LH}} \rightarrow \omega_{\mathrm{O}}$. Taking a limit as $m_{e} \rightarrow 0$ we obtain from (14)

$$
\left\{\frac{d^{2}}{d x^{2}}-k_{y}^{2}-\frac{\alpha \omega^{2} k_{y} \epsilon_{\mathrm{n}}}{\left(\omega^{2}-\omega_{\mathrm{O}}^{2}\right)\left(\omega-k_{y} V_{\mathrm{E}}(x)\right)}\right\} \tilde{\phi}_{1}(x)=0
$$

where $\alpha=\omega_{0}^{2} / \Omega_{0}$. The free energy for the EIH in this limit comes from coupling of the density gradient $\epsilon_{\mathrm{n}}$ with the sheared velocity $V_{\mathrm{E}}(x)$. Waves are produced with $\omega \sim \omega_{\mathrm{O}}$ and $k_{y} L_{\mathrm{E}} \sim 1$ ( $L_{\mathrm{E}}$, the flow velocity gradient scale length). Derivations of (14) and (15) are provided in the appendix.

To consider the development of the EIH instability in the depletion boundary during the simulation, we

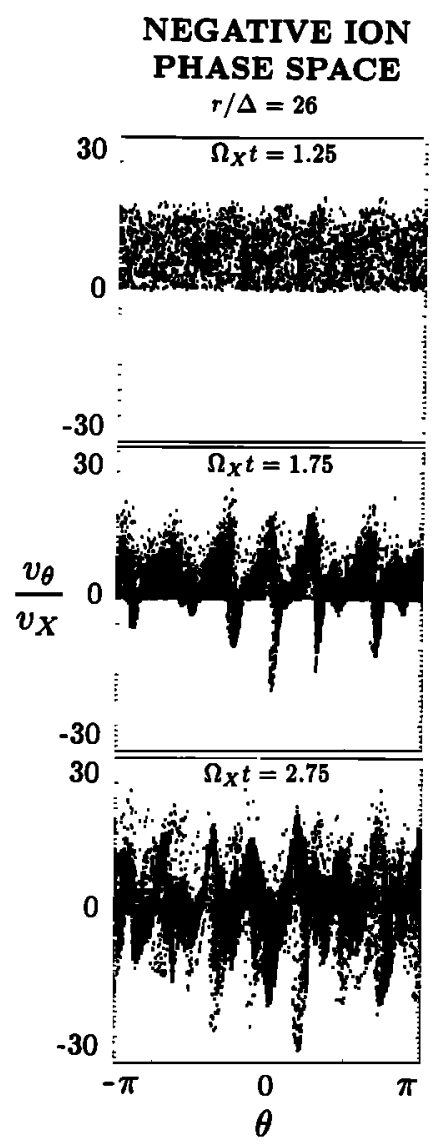

Figure 11. Azimuthal phase space of negative ions at $\Omega_{\mathrm{X}} t=1.25,1.75$, and 2.75 . The radius shown is $r / \Delta$ $=26$ to correspond to cross sections shown in Figures 8 and 9 . Note trapping of negative ions. simplify (15) by making a local approximation where $\tilde{\phi}_{1}(x)=\delta \phi \exp \left(i k_{x} x\right)$ and $k_{x}$ is the wavenumber in the $\hat{\mathbf{x}}$ direction. This implies $d^{2} / d x^{2} \rightarrow-k_{x}^{2}$ in (15). Also, since $V_{\mathrm{E}}$ is $\hat{\mathbf{y}}$ directed, the maximum growth occurs for $k_{x}=0$. Using these approximations, we obtain the local dispersion relation

$$
\tilde{\omega}^{3}+\left(\frac{\delta_{i} S}{\tilde{k}_{y}}-\frac{\alpha_{i}}{\delta_{i}} \tilde{k}_{y}\right) \tilde{\omega}^{2}-\tilde{\omega}+\frac{\alpha_{i}}{\delta_{i}} \tilde{k}_{y}=0
$$

where $\tilde{\omega}=\omega / \omega_{\mathrm{O}}$ and $\tilde{k}_{y}=k_{y} L_{\mathrm{E}}$ are the normalized frequency and wavenumber. Also, $\delta_{i}=\omega_{\mathrm{O}} / \Omega_{\mathrm{O}}$, $\alpha_{i}=V_{\mathrm{E}} /\left(\Omega_{\mathrm{O}} L_{\mathrm{E}}\right)$, and $S=L_{\mathrm{E}} / L_{\mathrm{n}}$. The ratio $\delta_{i}$ during the simulation is approximately 3.5. Early in the simulation when $\Omega_{\mathrm{x}} t \lessgtr 0.25$, we find $S \approx 1\left(L_{\mathrm{E}} \approx L_{\mathrm{n}} \approx 2 \Delta\right)$ and $\alpha_{i} \approx 3.2$. The numerical solution to $(16)$ for these parameters is shown in Figure 12. The solid line corresponds to the real part of the frequency $\omega_{r}$ and the dotted line corresponds to the imaginary part $\omega_{i}$ (growth rate). Maximum growth occurs for $k_{y} L_{\mathrm{E}} \approx 2$. The maximum growth rate and frequency at maximum growth are both approximately $0.8 \omega_{0}$.

We find that the linear growth phase for the waves in the boundary layer occurs very early in the simulation when $0.0<\Omega_{X} t \leqslant 0.125$. After this time period, the negative ion azimuthal phase space exhibits strong perturbations indicative of nonlinear processes. Using the azimuthal electrostatic field energy at the boundary, we estimate $\omega_{i} \approx 0.5 \omega_{0}$ during this time period. The wavelength $\lambda\left(2 \pi / k_{y}\right)$ may be estimated by assuming $\lambda=2 \pi r_{d} / m$, where $r_{d}$ is the depletion radius and $m$ is the mode number for perturbations in the boundary. During the linear phase, $r_{\mathrm{d}} \approx 6 \Delta$ and from Figure $6, m=6$. This implies $k_{y} L_{\mathrm{E}} \approx 2$. The wave frequency $\omega_{r}$ may be estimated from the phase velocity $v_{\phi}$ $\left(\omega_{r} / k_{y}\right)$ inferred from perturbations in the negative ion phase space. We estimate $v_{\phi} \approx-15 v_{\mathrm{X}}$ which implies $\omega_{r} \approx 0.5 \omega_{0}$. Therefore we find that the linear theory described by (16) provides a good description of the waves early in the simulation.

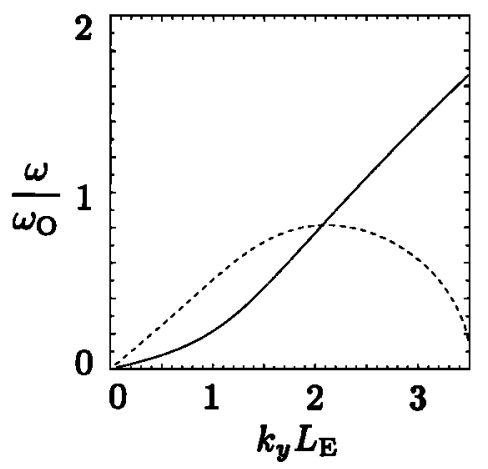

Figure 12. Dispersion relation ( $\omega$ versus $k_{y}$ ) showing the linear growth of the EIH instability early in the simulation. The solid line indicates real frequency, while the dotted line indicates imaginary frequency (growth rate). 


\section{Summary and Discussion}

We have presented results of a numerical simulation study to further understand physical processes associated with early time creation and evolution of electron depletions produced by attachment chemical releases. This work provides an important advance over our past work [Scales et al., 1992, 1994a] which did not include attachment chemistry. We find that many of the processes observed are qualitatively consistent with the early work, but the more realistic model shows several important fundamental differences. As in our earlier work, we find that a highly sheared electron flow velocity develops in the boundary of the depletion. However, since the attachment chemistry sustains the steep density gradients and negative charge surplus at the boundary, this sheared velocity flow is maintained for longer time periods. Unstable azimuthal waves develop due to this sheared velocity and density gradients at the boundary as in our earlier work. These waves are allowed to grow to larger amplitudes when chemistry is included. The waves produce plasma density irregularities in the depletion boundary. The electron density exhibits irregularities in the form of deep cavities and spikes where the size is smaller than the negative ion Larmor radius. This irregularity signature is dependent on the position in the boundary layer. As pointed out by Scales et al. [1994b], these show similarities with the irregularities observed during recent experiments [Bernhardt et al., 1994]. Irregularity development in the ions was observed with structuring in the negative ion density the most pronounced. This was not predicted by the earlier work. The negative ion cloud produced exhibits structures in the form of density clumps and spikes that are coincident with the electron density irregularities. Turbulent wave-particle heating of the negative ions is also observed. The positive ions are observed to receive negligible heating. Negative ion trapping becomes a more important mechanism for saturating the wave growth than the broadening of the electron flow velocity and density profiles that was described in our earlier work. All of the processes observed in our simulation model are expected to occur on a time period less than the heavy negative ion cyclotron period ( $0.1 \mathrm{~s})$.

Our results have important implications for the use of attachment chemical releases to produce artificial electron depletions and negative ion plasmas in the ionosphere. Particularly important is the development of plasma density irregularities during the early time evolution as is observed in our simulation results. These irregularities initially leave the plasma highly structured and therefore affect the creation and evolution over larger timescales. This fact has not been considered in past work and will be studied in our future modeling efforts. As we noted earlier, one important future use of artificially created electron depletions is the focusing of high-power radio waves [Bernhardt et al., 1994]. The deep electron density cavities and spikes in the depletion boundary produced by plasma instabilities during the release may have fundamental effects on the radio beam. This is an important problem that should be considered in detail with future investigations to futher understand focusing by artificially produced electron depletions.

\section{Appendix: Electron-Ion-Hybrid Instability}

In this Appendix we briefly outline the derivation of the eigenvalue conditions (14) and (15) which determine the stability of the depletion boundary layer. To obtain this result, the previous formalism of Ganguli et al. [1988] and Romero et al. [1992a] is generalized to include a negative ion species. In this formalism, the potential $\phi$, species density $n_{\mathbf{s}}$, and flow velocity $v_{\mathbf{s}}$ are linearized as follows:

$$
\begin{gathered}
\phi=\phi_{0}+\phi_{1}=\phi_{0}(x)+\tilde{\phi}_{1}(x) e^{i\left(k_{y} y-\omega t\right)} \\
n_{\mathrm{s}}=n_{\mathrm{s} 0}+n_{\mathrm{s} 1}=n_{\mathrm{s} 0}(x)+\tilde{n}_{\mathrm{s} 1}(x) e^{i\left(k_{y} y-\omega t\right)} \\
\mathbf{v}_{\mathrm{s}}=\mathbf{v}_{\mathrm{s} 0}+\mathbf{v}_{\mathrm{B} 1}=\mathbf{v}_{\mathrm{s} 0}(x)+\tilde{v}_{\mathrm{s} 1}(x) e^{i\left(k_{\mathrm{y}} y-\omega t\right)}
\end{gathered}
$$

Here subscripts 0 correspond to zeroth-order equilibrium quantities and 1 to first-order perturbed quantities. The magnetic field $\mathbf{B}$ is in the $\hat{\mathbf{z}}$ direction, $\hat{\mathbf{x}}$ denotes the direction of density and flow velocity gradients, and $\hat{\mathbf{y}}$ is the direction of the flow velocity and wave propagation. Note that $V_{\mathrm{E}}(x) \hat{\mathbf{y}}=\left(-\nabla \phi_{0}(x) \times \hat{\mathbf{z}}\right) / B$ is the sheared $\mathbf{E} \times \mathbf{B}$ flow velocity. From a Vlasov analysis of equilibrium features of the boundary layer [Ganguli et al., 1992], we find that the electrons are well magnetized since their shear frequency $\omega_{s}$ is much smaller than their cyclotron frequency $\Omega_{\text {ce }}$ (i.e., $\alpha_{e}=\omega_{\mathrm{s}} / \Omega_{\mathrm{ce}} \ll 1$, where $\omega_{\mathrm{B}}=\mathrm{V}_{\max } / L_{\mathrm{E}}, \mathrm{V}_{\max }$ is the peak value of the electron cross-field flow, and $L_{\mathrm{E}}$ is the characteristic scale-size of this flow). On the other hand, the ion shear frequencies are much larger than their cyclotron frequencies. This makes the ions effectively unmagnetized. We also find that the ion cross-field flows are negligible compared to the electron cross-field flow. Using these approximations in the linearized continuity and momentum equations [Ganguli et al., 1988; Romero et al., 1992a], the perturbed densities can be calculated to be

$$
\begin{gathered}
\tilde{n}_{e 1}(x)= \\
-\frac{\epsilon_{0} \delta^{2}}{q_{e}}\left\{-\frac{d^{2}}{d x^{2}}+k_{y}^{2}-\frac{k_{y}\left(V_{\mathrm{E}}^{\prime \prime}(x)-\epsilon_{\mathrm{n}} \Omega_{\mathrm{ce}}\right)}{\left(\omega-k_{y} V_{\mathrm{E}}(x)\right)}\right\} \tilde{\phi}_{1}(x) \\
\tilde{n}_{01}(x)=\frac{\epsilon_{0}}{q_{\mathrm{O}}} \frac{\omega_{\mathrm{O}}^{2}}{\omega^{2}}\left\{-\frac{d^{2}}{d x^{2}}+k_{y}^{2}\right\} \tilde{\phi}_{1}(x) \\
\tilde{n}_{\mathrm{X} 1}(x)=\frac{\epsilon_{0}}{q_{\mathrm{X}}} \frac{\omega_{\mathrm{X}}^{2}}{\omega^{2}}\left\{-\frac{d^{2}}{d x^{2}}+k_{y}^{2}\right\} \tilde{\phi}_{1}(x)
\end{gathered}
$$

Here $\omega_{\mathrm{O}}, q_{\mathrm{O}}$ and $\omega_{\mathrm{X}}, q_{\mathrm{X}}$ are the plasma frequency and charges for the positive and negative ions, respectively, $V_{\mathrm{E}}^{\prime \prime}=d^{2} V_{\mathrm{E}} / d x^{2}$, and $\delta=\omega_{\mathrm{pe}} / \Omega_{\mathrm{ce}}$. The permittivity constant is $\epsilon_{0}$ and $\epsilon_{\mathrm{n}}=\left(d n_{e 0} / d x\right) / n_{e 0}$ is the density gradient inverse scale length. Using the above expressions 
along with the Poisson equation $\left(-\epsilon_{0} \nabla^{2} \phi_{1}=\Sigma_{\mathrm{s}} q_{\mathrm{s}} n_{\mathrm{s} 1}\right)$, we obtain

$$
\begin{aligned}
\left\{1-\frac{\omega_{\mathrm{O}}^{2}}{\omega^{2}}-\frac{\omega_{\mathrm{X}}^{2}}{\omega^{2}}+\delta^{2}\right\}\left\{-\frac{d^{2}}{d x^{2}}+k_{y}^{2}\right\} \tilde{\phi}_{1}(x)- \\
\delta^{2} \frac{k_{y}\left(\mathrm{~V}_{\mathrm{E}}^{\prime \prime}-\epsilon_{\mathrm{n}} \Omega_{\mathrm{ce}}\right)}{\omega-k_{y} \mathrm{~V}_{E}} \tilde{\phi}_{1}(x)=0
\end{aligned}
$$

which can be rewritten as

$$
\left\{\frac{d^{2}}{d x^{2}}-k_{y}^{2}-G(\omega) \frac{k_{y}\left(V_{\mathrm{E}}^{\prime \prime}(x)-\epsilon_{\mathrm{n}} \Omega_{\mathrm{ce}}\right)}{\omega-k_{y} V_{\mathrm{E}}(x)}\right\} \tilde{\phi}_{1}(x)=0
$$

where

$$
\begin{gathered}
G(\omega)=\frac{\delta^{2}}{\left(\delta^{2}+1\right)\left(1-\left(\omega_{\mathrm{LH}} / \omega\right)^{2}\right)} \\
\omega_{\mathrm{LH}}^{2}=\frac{\omega_{\mathrm{O}}^{2}+\omega_{\mathrm{X}}^{2}}{1+\left(\omega_{\mathrm{pe}} / \Omega_{\mathrm{ce}}\right)^{2}}
\end{gathered}
$$

is the lower hybrid frequency which takes into account the contribution from both ion species. It should be noted that in general $m_{\mathrm{X}} \gg m_{\mathrm{O}}$, so $\omega_{\mathrm{O}}^{2} \gg \omega_{\mathrm{X}}^{2}$ and $\omega_{\mathrm{LH}}$ is close to the lower hybrid frequency of the background $\mathrm{O}^{+}$plasma. It can also be noted that the eigenvalue condition in (A8) is identical to the work of Ganguli et al. [1988] and Romero et al. [1992a] except for the inclusion of the negative ion plasma frequency contribution in $\omega_{\mathrm{LH}}$. Since these works provide detailed studies of the characteristics of the EIH instability, they will not be repeated here and we refer the reader to these works for details.

For massless, cold electrons, the momentum equation for the electrons becomes altered from the work of Ganguli et al. [1988] and Romero et al. [1992a]. In this case, the momentum equation becomes

$$
\mathbf{v}_{e}=\frac{\mathbf{E} \times \mathbf{B}}{B^{2}}
$$

Linearizing this form of the momentum equation with the continuity equation (which remains the same) yields the perturbed electron density

$$
\tilde{n}_{e 1}(x)=-\frac{\epsilon_{0}}{q_{e}} \frac{\left(\omega_{\mathrm{O}}^{2} / \Omega_{\mathrm{O}}\right) k_{y} \epsilon_{\mathrm{n}}}{\left(\omega-k_{y} V_{\mathrm{E}}(x)\right)} \tilde{\phi}_{1}(x)
$$

Using this result with the perturbed ion densities (A5) and (A6) in the Poisson equation yields (15). Note in (15) that the $\omega_{X}^{2}$ term has been dropped since $\omega_{\mathrm{O}}^{2} \gg$ $\omega_{\mathrm{X}}^{2}$. As stated in the text, taking the limit as $m_{e} \rightarrow 0$ on (14) yields (15) as might be expected. Since the electrons are massless in this limit, the waves are no longer of lower hybrid type but correspond to positive ion plasma oscillations. The EIH instability is driven by the coupling between the electron density gradient $\epsilon_{\mathrm{n}}$ and the sheared flow velocity $V_{\mathrm{E}}$ in this regime.

Acknowledgments. This work was supported at VA Tech by ONR grants N00014-92-J-1484 and N00014-92-J1498. The work was supported at NRL by NASA and ONR.

The Editor thanks R. A. Roussel-Dupre' and W. J. Raitt for their assistance in evaluating this paper.

\section{References}

Bernhardt, P. A., Chemistry and dynamics of $S F_{6}$ injections into the $F$ region, $J$. Geophys. Res., 89, 3929, 1984.

Bernhardt, P. A., A critical comparison of ionospheric depletion chemicals, J. Geophys. Res., 92, 4617, 1987.

Bernhardt, P. A., Cross-B convection of artificially created, negative ion clouds and plasma depressions: Low-speed flow, J. Geophys. Res., 93, 8696, 1988.

Bernhardt, P. A., and L. M. Duncan, Ionospheric focused heating - a hybrid modification experiment, J. Atmos. Terr. Phys., 49, 1107, 1987.

Bernhardt, P. A., E. J. Weber, J. G. Moore, J. Baumgardner, and M. Mendillo, Excitation of oxygen permitted line emissions by $S F_{6}$ injections into the $F$ region, $J$. Geophys. Res., 91, 8937, 1986.

Bernhardt, P. A., R. A. Roussel-Dupree, M. B. Pongratz, G. Haerendel, A. Valenzuela, D. A. Gurnett, and R. R. Anderson, Observations and theory of the AMPTE magnetotail barium releases, J. Geophys. Res., 92, 5777, 1987.

Bernhardt, P. A., P. Rodriquez, C. L. Siefering, and C. S. Lin, Field-aligned dynamics of chemically induced perturbations to the ionosphere, J. Geophys. Res., 96, 13887, 1991.

Bernhardt, P. A., J. D. Huba, J. A. Fulford, P. A. Forsyth, D. N. Anderson, and S. T. Zalesak, Analysis of rocket beacon transmissions to reconstruct ionospheric densities, Radio Sci., 28, 613, 1993.

Bernhardt, P. A., et al., The ionospheric focused heating experiment, J. Geophys. Res., in press, 1994.

Birdsall, C. K., and A. B. Langdon, Plasma Physics Via Computer Simulation, Adam Hilger, New York, 1991.

Ganguli, G., Y. C. Lee, and P. Palmadesso, Electron-ion hybrid mode due to transverse velocity shear, Phys. Fluids, $31,2753,1988$.

Ganguli, G., P. A. Bernhardt, W. Scales, P. Rodriquez, C. L. Siefering, and H. Romero, Physics of negative ion plasmas created by chemical releases in space, in Physics of Space Plasmas (1992), SPI Conf. Proc. Reprint Ser., vol. 12, edited by $T$. Chang and J. Jasperse, p. 161, Scientific, Cambridge, Mass., 1992.

Gottlieb, D., and S. A. Orszag, Numerical Analysis of Spectral Methods: Theory and Applications, SIAM-CBMS, Philadelphia, Pa., 1977.

Hasegawa, A., Plasma Instabilities and Nonlinear Effects, Springer-Verlag, New York, 1975.

Hockney, R. W., and J. W. Eastwood, Computer Simulation Using Particles, Adam Hilger, New York, 1988.

Huba, J. D., A. B. Hassam, and D. Winske, Stability of sub-Alfvenic plasma expansions, Phys. Fluids $B, 2,1676$, 1990.

Orszag, S. A., Numerical simulation of incompressible flows within simple boundaries, 1, Galerkin (spectral) methods, Stud. Appl. Math., L, 293, 1971.

Mendillo, M., and D. Forbes, Theory and observations of a dynamically evolving negative ion plasma, J. Geophys. Res., 87, 8273, 1982.

Patterson, G. S., and S. A. Orszag, Spectral calculations of isotropic turbulence: Efficient removal of aliasing interactions, Phys. Fluids, 14, 2538, 1971.

Romero, H., G. Ganguli, Y. C. Lee, and P. J. Palmadesso, Electron-ion hybrid instabilities driven by velocity shear in a magnetized plasma, Phys. Fluids B, 4, 1708, 1992a.

Romero, H., G. Ganguli, and Y. C. Lee, Ion acceleration and coherent structures generated by lower hybrid sheardriven instabilities, Phys. Rev. Lett., 69, 3503, 1992b.

Scales, W. A., and P. A. Bernhardt, Simulation of highspeed (orbital) releases of electron attachment materials in the ionosphere, J. Geophys. Res., 96, 13,815, 1991. 
Scales, W. A., P. A. Bernhardt and G. Ganguli, Numerical simulation of the evolution of negative ion clouds produced during electron attachment chemical release experiments, in Physics of Space Plasmas (1992), SPI Conf. Proc. Reprint Ser., vol. 12, edited by T. Chang and J. Jasperse, p. 597, Scientific, Cambridge, Mass., 1992.

Scales, W. A., P. A. Bernhardt and G. Ganguli, Early time evolution of negative ion clouds and electron density depletions produced during electron attachment chemical release experiments, J. Geophys. Res., 99, 373, 1994 a.

Scales, W. A., P. A. Bernhardt, G. Ganguli, C. L. Siefring, and $P$. Rodriguez, Small-scale plasma irregularities produced during electron attachment chemical releases, Geophys. Res. Lett., 21, 605, 1994 b.

Tajima, T., Computational Plasma Physics: With Applications to Fusion and Astrophysics, Addison Wesley, New York, 1989.
Winske, D., Development of flute modes on expanding plasma clouds, Phys. Fluids B, 1, 1900, 1989.

P. A. Bernhardt and G. Ganguli, Beam Physics Branch, Plasma Physics Division, Naval Research Laboratory, Washington DC 20375-5000. (e-mail: bern@ppd.nrl.navy.mil; gang@ppd.nrl.navy.mil)

W. A. Scales, Bradley Department of Electrical Engineering, Virginia Tech, Blacksburg, VA 24061-0111. (e-mail: wayne@starchild.ee.vt.edu)

(Received March 14, 1994; revised September 1, 1994; accepted September 19, 1994.) 